\title{
Corticosteroid-induced ocular hypertension. II. An acquired form
}

SUMMARY Thirty-five patients with unilateral closed-angle glaucoma treated by peripheral iridectomy and prophylactic peripheral iridectomy in the fellow eyes were subjected to corticosteroid provocative test in both eyes. $51 \%$ of the eyes with closed-angle glaucoma and $11 \%$ of their fellow eyes had a positive corticosteroid pressure response. The closed-angle glaucoma eyes and their fellow eyes responded differently as shown by the frequency distribution graphs and also by the difference between the corticosteroid-induced change in pressure (Wilcoxon test, $z=-4 \cdot 80$, $p<0.0001$ ). These results provide evidence for an acquired form of corticosteroid-induced ocular hypertension and the possible pathogenic factors for the first time. The clinical significance of an acquired form of corticosteroid-induced ocular hypertension is discussed.

Ever since the conception of corticosteroid-induced ocular hypertension was advanced by François' in 1954 the subject has $\cdot$ been extensively studied and widely reported. In the 2 decades following François's paper reports on further observations, ideas on pathogenesis, the genetic influence, clinical significance, and the prognostic and investigative value of the corticosteroid pressure response have appeared. ${ }^{2-10}$ Notable among these early reports were those by Armaly $\rightarrow$ and by Becker and coworkers, ${ }^{10-12}$ which led to the hypothesis that corticosteroid-induced ocular hypertension was inherited as a single-gene autosomal trait and that it was intimately related to the inheritance of primary open-angle glaucoma. This basic premise, if correct, was supposed to transform the corticosteroid provocative test into a valuable predictive and diagnostic tool in screening patients for primary open-angle glaucoma. It would also help to select those glaucoma suspects who require treatment because of a definite risk of progression to glaucoma. The potential of such a test and its status in clinical ophthalmology were never realised, and other observers started to question the validity of the monogenic hypothesis. ${ }^{31314}$

Evidence to support an acquired form of corticosteroid-induced ocular hypertension is here presented. The possible pathogenic factors in the production of such an acquired response are identified and discussed.

\section{Material and methods}

A corticosteroid provocative test, as described in the first part of this paper, was performed on patients attending the outpatient clinics who had sustained a spontaneous episode of closed-angle glaucoma in only one eye and been treated initially with a peripheral iridectomy with or without supplementary medical therapy. All these patients had been operated on at least six months previously, and those on supplementary medical therapy had been on the same treatment for at least 6 months without any change and with normal intraocular pressures. The fellow eyes in all these patients showed no evidence of a previous episode of closed-angle glaucoma, though 5 of them had a positive phenylephrine-pilocarpine provocative test (nos. 8, 16, 19, 24, 35). All the fellow eyes had been subjected to prophylactic peripheral iridectomies.

Patients excluded from this study include (a) those on systemic steroids or carbonic anhydrase inhibitors; (b) those who proceeded to have a drainage operation following the initial peripheral iridectomy.

The sample consisted of 35 patients, all Caucasians, 8 males and 27 females, aged 49-80 years (average 68.5 years, SD 8.22). The episode of closed-angle glaucoma occurred in the right eye in 18 patients and in the left eye in 17. Six patients were on supplementary medical therapy. Sixteen had significant peripheral anterior synechiae ranging from $10 \%$ to $60 \%$ (mean $26 \%$, SEM $3 \cdot 23$ ), and 8 had a significant amount of pigment in the angle.

\section{Results}

The corticosteroid-induced pressure changes in the eyes with closed-angle glaucoma and their fellow eyes are shown in Table $8.51 \%$ (18 out of 35) of the closed-angle glaucoma eyes responded to the topical 
Table 8 Results of corticosteroid provocative testing

\begin{tabular}{|c|c|c|c|c|c|}
\hline & \multicolumn{2}{|c|}{ Close-angle glaucoma eyes (CAGE) } & \multicolumn{2}{|c|}{ Fellow eyes $(F E)$} & $\triangle P C A G E-\triangle P F E$ \\
\hline $\begin{array}{l}\text { Mean } \\
\text { SEM }\end{array}$ & $\begin{array}{r}+7.26 \\
1.09\end{array}$ & $\begin{array}{r}-0.02 \\
0.02\end{array}$ & $\begin{array}{r}+2.43 \\
0.54\end{array}$ & $\begin{array}{r}-0.02 \\
0.02\end{array}$ & $\begin{array}{r}+4.83 \\
0.81\end{array}$ \\
\hline
\end{tabular}

$\Delta P=$ Change in pressure $(\mathrm{mmHg}) . \Delta C=$ change in outflow facility. SEM=standard error of the mean.

dexamethasone with a change in pressure greater than $5 \mathrm{mmHg}$, whereas only $11 \%$ (4) of their fellow eyes had a change in pressure greater than $5 \mathrm{mmHg}$. The 4 patients with a positive response in the fellow eyes also showed the greatest response in their closedangle glaucoma eyes.

Figs. 1 and 2 show the frequency distributions of the intraocular pressures in the eyes with closed-angle glaucoma and the fellow eyes respectively, before and after the corticosteroid provocative testing. The distributions of the initial pressures (pretest) in both groups of eyes were near normal. The mean initial pressure in the closed-angle glaucoma eyes was $18 \cdot 11$ mmHg (SEM 0.54, variance 10.04). The mean initial pressure for the fellow eyes was $17.03 \mathrm{mmHg}$ (SEM $0 \cdot 47$, variance $7 \cdot 46$ ).

The distribution of the post-test intraocular pressure (final pressure) in the fellow eyes retained the general outline of a Gaussian curve but with a positive skew (Fig. 2). A more representative picture is obtained by using change in pressure as the

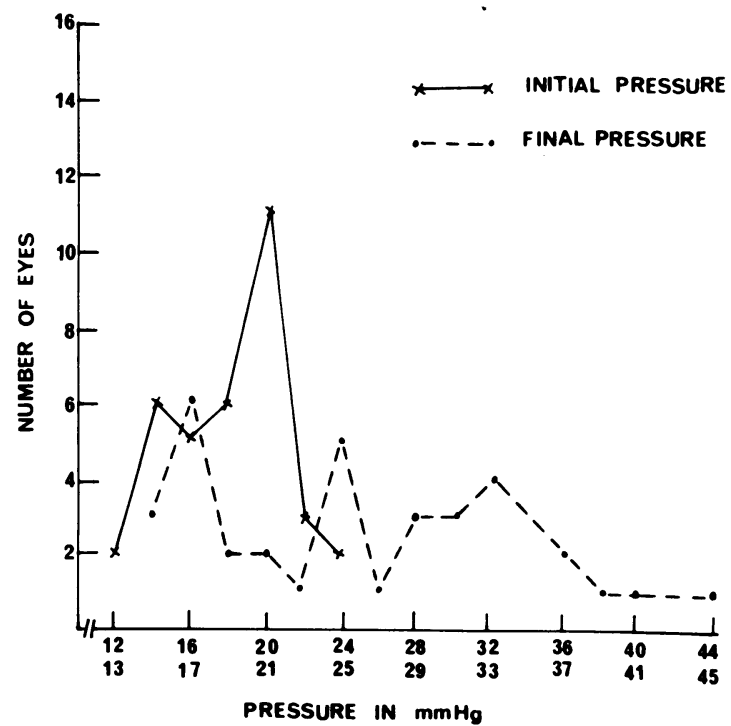

Fig. 1 The frequency distributions of the initial pressures and the final pressures following corticosteroid provocative tests in eyes with closed-angle glaucoma. parameter for corticosteroid-induced ocular hypertension, and Fig. 3 illustrates the frequency distribution of the corticosteroid-induced pressure change in the fellow eyes. The distribution remained near Gaussian with a positive skew.

The frequency distribution of the final pressures in the eyes with closed-angle glaucoma is shown in Fig. 1 and the frequency distribution of the corticosteroidinduced change in pressure in the same eyes is illustrated by Fig. 4.

A final graphic analysis is employed to compare and contrast the frequency distributions of the corticosteroid-induced pressure change in the fellow eyes and the eyes with closed-angle glaucoma and also to assess the conformity of the observed distributions already (Figs. 3 and 4 respectively) to a Gaussian or multimodal distribution. Figs. 5 and 6 illustrate the percentage cumulative frequency plotted along a probability ordinate scale constructed so that a Gaussian distribution would appear linear. The percentage cumulative frequency distribution of

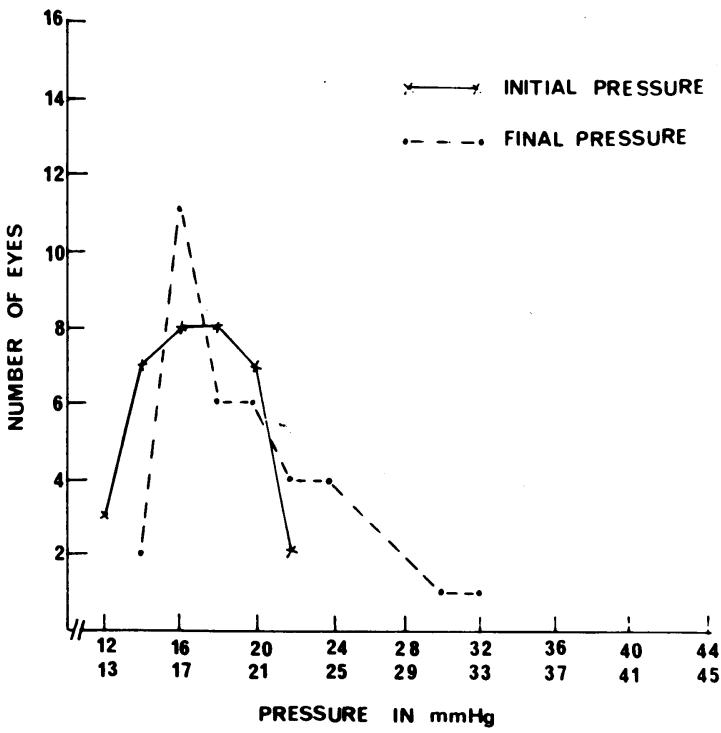

Fig. 2 The frequency distribution of the initial pressures and final pressures following corticosteroid provocative tests in the fellow eyes. 


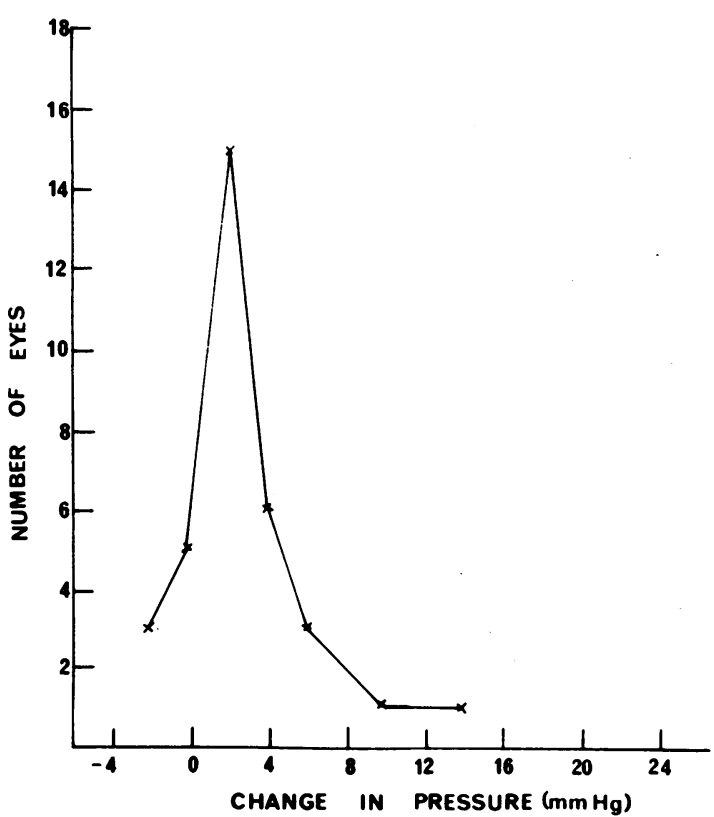

Fig. 3 Frequency distribution of corticosteroid-induced change in pressure in fellow eyes.

the corticosteroid-induced changes in pressure in the fellow eyes is shown in Fig. 5. The graph is curvilinear, implying that the distribution of the pressure change in the fellow eyes is not a simple Gaussian curve, but it distinctly lacks the inflections which would suggest multimodality. The corresponding graph for the eyes with closed-angle glaucoma (Fig. 6) on the other hand shows these inflections rather convincingly.

The difference between the corticosteroid pressure response of the paired eyes of each patient (one closed-angle glaucoma eye and its clinically normal fellow eye) is calculated and shown in the last column of Table 1 . There is a highly significant statistical difference between the corticosteroid-induced change in pressure in the matched pairs of eyes (Wilcoxon test, $\mathrm{z}=-4.80, \mathrm{p}<0.0001$ ). The difference in change in pressure in each pair is greater than $5 \mathrm{mmHg}$ in $46 \%$ of the patients.

\section{Discussion}

The mean initial pressure for the eyes with closedangle glaucoma $(18.11 \mathrm{mmHg})$ is significantly higher $(p=0.02, t$ test) than the mean initial pressure for the fellow eyes $(17.03 \mathrm{mmHg})$, but there is no significant difference in the relative dispersion of the initial pressures in the 2 groups ( $>>0.05, F$ test). This implies that the closed-angle glaucoma eyes and their fellow eyes before the corticosteroid provocative test constitute a homogeneous population, that is, the 2

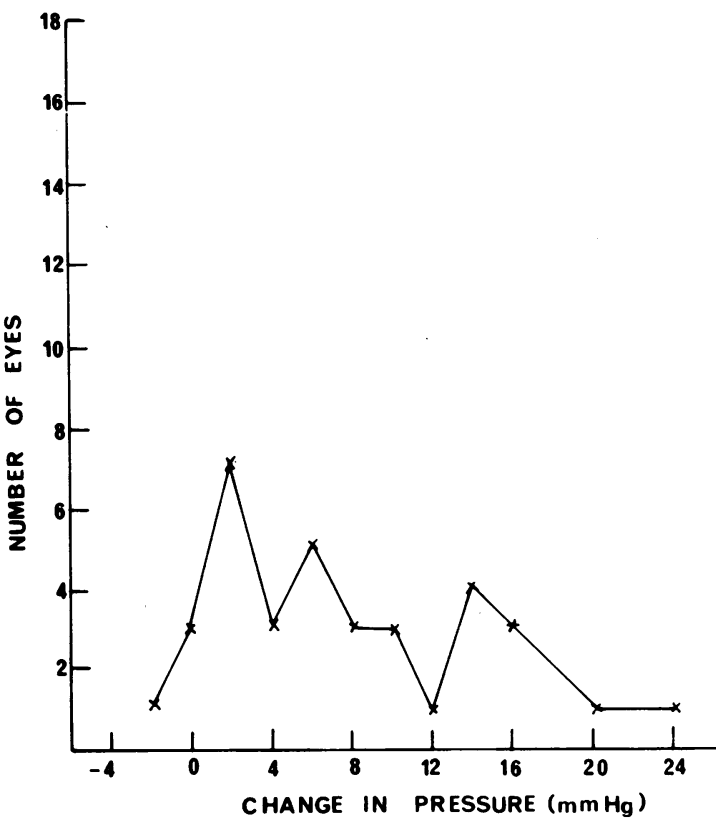

Fig. 4 Frequency distribution of corticosteroid-induced change in pressure in closed-angle glaucoma eyes.

samples are from the same population. The fact that clinically normal eyes after an attack of closed-angle glaucoma show a significant increase in intraocular pressure relative to the fellow eyes was reported by Mapstone. ${ }^{15}$

Because the number of patients in the sample in this study is relatively small, the interpretation of the graphic illustrations must be made with caution. Firstly, the percentage cumulative frequency distribution of the change in pressure in the fellow eye (Fig. 5) does not show the multimodality expected with the hypothesis of simple monogenic inheritance as proposed by Armaly ${ }^{7 \rightarrow}$ and Becker et al. ${ }^{10-12}$ Secondly, the frequency distribution of the final pressures in the closed-angle glaucoma eyes (Fig. 1) and the frequency distribution of the corticosteroidinduced pressure change in the eyes with closed-angle glaucoma (Fig. 4) are different from the corresponding graphs for the fellow eyes in 2 ways: $(a)$ there is a much wider scatter of the abscissa pointsfor example, the final pressures have a variance of $64 \cdot 12$ compared with $16 \cdot 48$ for the fellow eyes; $(b)$ the 2 graphs appear to have more than a single mode. This is confirmed by the inflections in Fig. 6.

These results reinforce an earlier observation that eyes that have sustained a spontaneous episode of closed-angle glaucoma respond differently to topical corticosteroids when compared with clinically normal eyes, but also, by the use of matched pairs of eyes, they allow us to dismiss any influence the genotype of the 


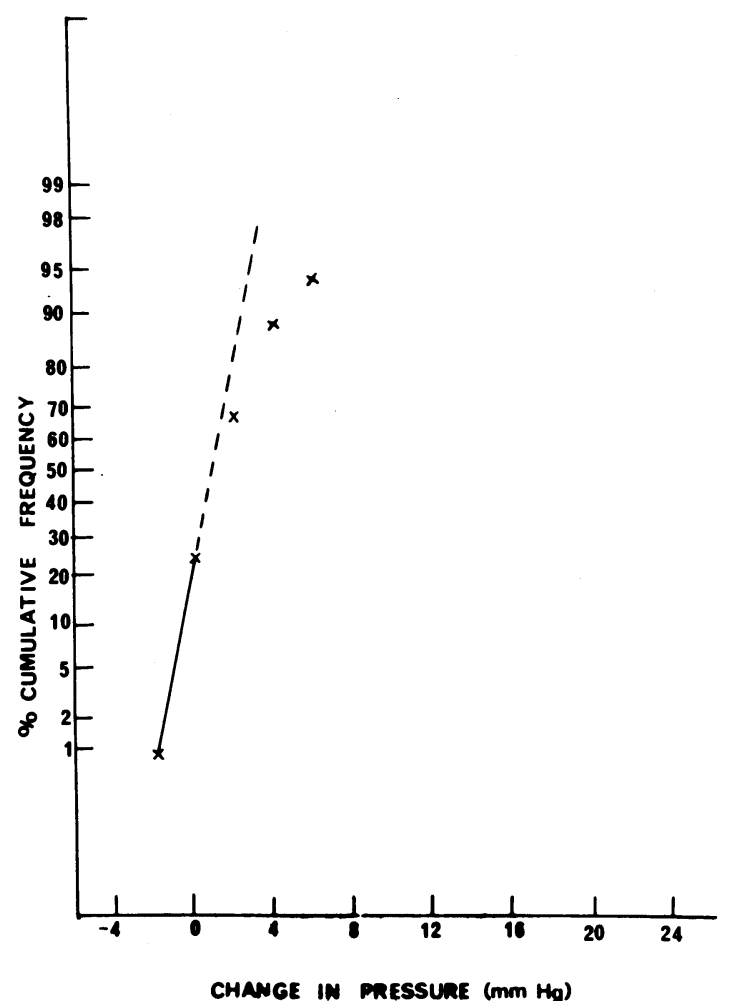

Fig. 5 Percentage cumulative frequency distribution of corticosteroid-induced change in pressure in fellow eyes.

patient might have on the corticosteroid-induced pressure response. The effects of other factors, such as patient motivation and compliance and time-delay effect on intraocular pressure, also become insignificant when the difference between the matched pair of eyes is considered. The logical deduction from this is that corticosteroid-induced ocular hypertension can be acquired by eyes that have sustained spontaneous episode of closed-angle glaucoma. Two possible explanations exist: (1) closed-angle glaucoma facilitates corticosteroid-induced ocular hypertension; (2) both closed-angle glaucoma and corticosteroid-induced ocular hypertension are due to a third common factor.

If one accepts that closed-angle glaucoma is a consequence of partial angle closure, then it is quite reasonable to suggest that partial angle closure could be the common factor to closed-angle glaucoma and corticosteroid-induced ocular hypertension. Partialangle glaucoma is also a recognised mechanism in the pathogenesis of some primary open-angle glaucoma and ocular hypertension. Both these groups of patients have a higher prevalence of corticosteroidinduced ocular hypertenson than normal persons,

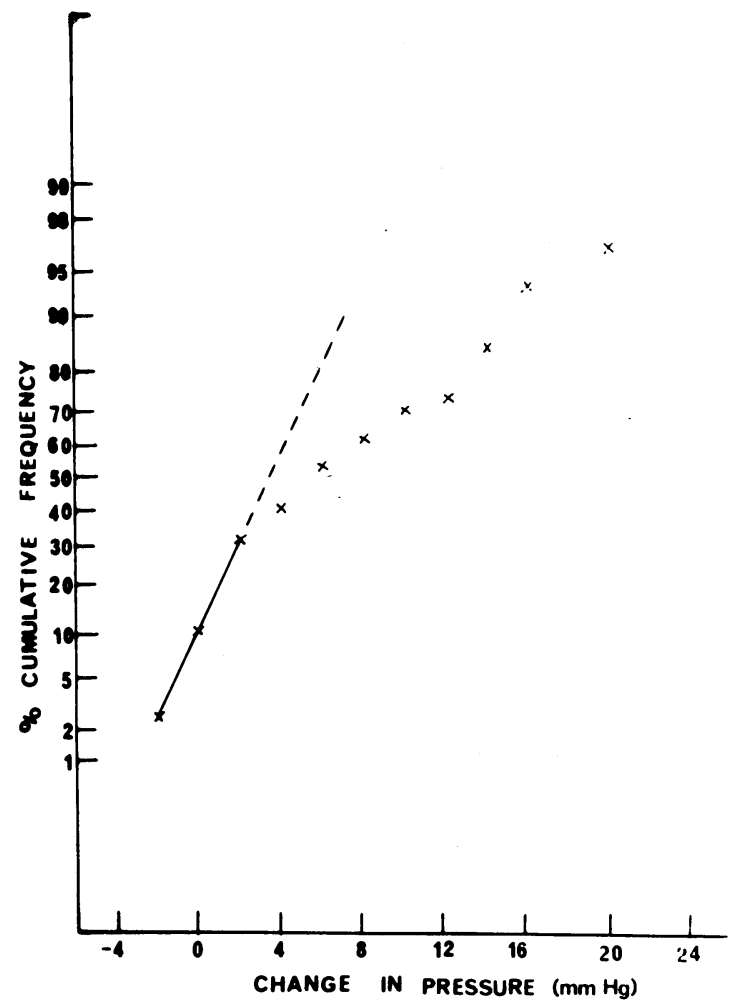

Fig. 6 Percentage cumulative frequency distribution of corticosteroid-induced change in pressure in eyes with closed-angle glaucoma.

and it seems logical to assume that the effect of the partial angle closure contributes to this higher prevalence.

Partial angle closure produces raised intraocular pressure, which inevitably leads to outflow damage. It would be interesting to identify which of the 3 factors, namely, partial angle closure, raised intraocular pressure, or damage to trabecular meshwork, is directly associated with corticosteroid-induced ocular hypertension. There is indeed no reason to presume that only one of these factors is involved; 2 or even all 3 could be.

There is evidence to suggest that the allele $\mathrm{p}^{\mathrm{H}}$ either predisposes individuals to the development of corticosteroid-induced ocular hypertension or directly influences the magnitude of the pressure response of individuals to topical corticosteroids. If the possession of this gene is offered as being responsible for the 4 fellow eyes with positive corticosteroid pressure response (patient nos. 3, 18, 28, 34), then one would expect their corresponding closedangle glaucoma eyes to have positive pressure responses. Not only do these 4 closed-angle glaucoma eyes have a positive corticosteroid pressure response 
but, as mentioned earlier, they show the highest change in pressure in the closed-angle glaucoma eyes. The implication of this is that the genetic influence and the effect of partial angle closure appear to be cumulative in the production of corticosteroidinduced ocular hypertension.

The interaction between genetic and acquired factors and other considerations make meaningless any sharp antithesis between genetically determined corticosteroid-induced ocular hypertension and that produced by acquired factors or environmental agents. There is in fact much overlap, and only in extreme cases can hereditary and acquired factors be clearly disentangled in the effect they produce.

In conclusion, corticosteroid-induced ocular hypertension does not appear to follow the simple Mendelian patterns of inheritance. It is suggested that the genetic factor in the aetiology is composed of one or more genes having a small, similar, or cumulative effect. The acquired factors acting on this genotype produce more of the phenotypic variations observed in the population than would be expected with a single gene trait. This pattern of inheritance has been termed multifactorial.

\section{Clinical significance}

The fact that corticosteroid-induced ocular hypertension can be acquired does not appear to be relevant to clinical practice at first. On further consideration the following points can be made:

1. A high corticosteroid pressure response is not a reliable indicator of future rising trend in intraocular pressure.

2. The role of the corticosteroid provocative test in the screening of individuals for glaucoma has to be revised.

3. Topical corticosteroids should be avoided in eyes with closed-angle glaucoma, especially during the postoperative period, when they are commonly prescribed. If there is an indication for their use, they should be restricted to one or 2 weeks, or the patient kept under closer observation. There is in fact evidence to suggest that the inflammatory activity in the anterior chamber after operation is not dramatically affected by topical corticosteroids (unpublished observation).

4. The fact that one eye in a patient does not respond to topical corticosteroid does not mean that the other eye will not respond.

I thank Mr R Mapstone for his inspiration and advice. I also thank the consultants who referred patients, Miss Sue Cowin for administrative assistance, Mr R. McBride for technical assistance, and Mrs M. D. Kelley for typing the paper.

\section{References}

1 François J. Cortisone et tension oculaire. Ann Oculist (Paris) 1954; 187: 805-16.

2 Goldman H. Cortisone glaucoma. Arch Ophthalmol 1961; 68: 621-6.

3 Godel V, Regenbogen L, Stein R. On the mechanism of corticosteroid-induced ocular hypertension. Ann Ophthalmol 1978; 10: 191-6.

4 Siliato F. Pathogenesis of corticosteroid-induced glaucoma. Minerva Oftalmol 1969; 11: 160-3.

5 François J. Corticosteroid glaucoma. Ann Ophthalmol 1977; 9: 1075-80.

6 Dean GO, Deutsch AR, Hiatt RL. The effect of dexamethasone on borderline ocular hypertension. Ann Ophthalmol 1975; 9: 193-8.

7 Armaly MF. The heritable nature of dexamethasone-induced ocular hypertension. Arch Ophthalmol 1966; 75: 32-6.

8 Armaly MF. Statistical attributes of the steroid hypertensive response in the clinically normal eye. I. The demonstration of three levels of response. Invest Ophthalmol Visual Sci 1965; 4: 187-97.

9 Armaly MF. Inheritance of dexamethasone hypertension and glaucoma. Arch Ophthalmol 1967; 77: 747-51.

10 Becker B. Hahn KA. Topical corticosteroids and heredity in primary open-angle glaucoma. Am J Ophthalmol 1964; 57: 543-51.

11 Becker B, Mills DW. Corticosteroids and intraocular pressure. Arch Ophthalmol 1963; 70: 500-7.

12 Becker B. Intraocular pressure response to topical corticosteroids. Invest Ophthalmol Visual Sci 1965; 4: 198-205.

13 Schwartz JT, Reuling FH, Feinleib M, Garrison RJ, Collie DJ. Twin study on ocular pressure after topical dexamethasone. I. Frequency distribution of pressure response. Am J Ophthalmol 1973; 76: 126-36.

14 Schwartz JT, Reuling FH, Feinleib M, Garrison RJ, Collie DJ. Twin study on ocular pressure following topically applied dexamethasone. II. Inheritance of variation in pressure response. Arch Ophthalmol 1973; 90: 281-6.

15 Mapstone R. Outflow changes in normal eyes after closed-angle glaucoma. Br J Ophthalmol 1977; 61: 637-40. 